\title{
Avaliação ecocardiográfica de equinos após exercício de polo ${ }^{1}$
}

\author{
Camila A.O. Bello², Cinthia B.S. Dumont ${ }^{2}$, Thaís C. Souza², Nina T. Caniello², Roberta F. Godoy², \\ José Renato J. Borges ${ }^{2}$, Meryonne Moreira² e Eduardo M.M. Lima ${ }^{2 *}$
}

\begin{abstract}
Bello C.A.O., Dumont C.B.S., Souza T.C., Caniello N.T., Godoy R.F., Borges J.R.J., Moreira M. \& Lima E.M.M. 2012. [Echocardiographic evaluation of horses after exercise polo.] Avaliação ecocardiográfica de equinos após exercício de polo. Pesquisa Veterinária Brasileira 32(11):1191-1195. Pós-Graduação em Saúde Animal, Faculdade de Agronomia e Medicina Veterinária, Universidade de Brasília, ICC Sul, Campus Universitário Darcy Ribeiro, Cx. Postal 4508, Brasília, DF 70910-970, Brazil. E-mail: limaemm@unb.br

Considering the complexity of the physical effort inherent in polo horses in competitions and the lack of reports about the cardiovascular effects resulting from a Chukker, yet the dynamics of the associated weight, the aim of this study was to evaluate by echocardiography with 27 horses polo athletes who underwent training starting at rest and after exercise. The results showed that this method has changed several echocardiographic indexes within five to ten minutes before the end of a Chukker. It was they, as structural index, thickness reduction interventricular septum and left ventricular free wall and increased left ventricular diameter, and functional as an increase in cardiac output and heart rate, while stroke volume, fractional shortening and ejection fraction reduced. The weight loss was not significant, so was not associated with echocardiographic changes. These results suggest that cardiac demand was high, indicating the importance of the physical and cardiovascular training specific to this modality.
\end{abstract}

INDEX TERMS: Cardiac function, echocadiography, polo horse.

RESUMO.- Considerando a complexidade do esforço físico inerente a cavalos em competições de polo e da carência de relatos na literatura sobre os efeitos cardíacos resultantes de um chukker, associado ainda a dinâmica do peso, o objetivo deste estudo foi de avaliar por meio da ecocardiografia, 27 equinos atletas de polo, submetidos à partida treino, em repouso e após o exercício. Os resultados demonstraram que esta modalidade alterou diversos índices ecocardiográfico no período de cinco a dez minutos do término de um chukker. Foram eles, estruturais com: redução da espessura do septo interventricular e parede livre do ventrículo esquerdo e aumento do diâmetro interno do ventrículo esquerdo em sístole, e funcionais como: aumento do débito cardíaco e frequência cardíaca, enquanto que o volume sistólico, a fração de encurtamento e a fração de ejeção reduziram. A perda de peso corporal não foi significativa,

\footnotetext{
${ }^{1}$ Recebido em 13 de março de 2012.

Aceito para publicação em 21 de junho de 2012.

${ }^{2}$ Programa de Pós-Graduação em Saúde Animal, Faculdade de Agronomia e Medicina Veterinária, Universidade de Brasília (UnB), ICC Sul, Campus Universitário Darcy Ribeiro, Cx. Postal 4508, Brasília, DF 70910-970, Brasil.

*Autor para correspondência: limaemm@unb.br
}

portanto não foi associado às alterações ecocardiográficas. Estes resultados sugeriram que a demanda cardíaca foi alta, indicando a importância de um acompanhamento físico e treinamento cardiovascular específico para esta modalidade.

TERMOS DE INDEXAÇ̃̃O: Função cardíaca, índices ecocardiográficos, cavalos de polo.

\section{INTRODUÇÃO}

0 conhecimento das exigências competitivas de diferentes esportes é importante para a concepção de programas de treinamento adequados. Assim, pode-se garantir que os animais alcancem um nível suficiente de condicionamento, reduzindo o risco de esforço excessivo e lesão, conseguindo melhora no desempenho em relação ao potencial genético individual. No que diz respeito à complexidade do desafio físico que cavalos de polo são submetidos, há poucas publicações sobre as demandas fisiológicas dos animais em relação a essa modalidade. Sendo que, em contrariedade a maioria dos esportes equestres, a demanda fisiológica de um jogo de polo é variável, pois se intercalam momentos de pausa, mudanças de velocidade e direção de movimentos, 
em um campo de grama de 275 x 180m, sendo cada partida composta por seis chukkers, ou seja, seis períodos de sete minutos, utilizando-se um animal diferente para cada chukker. ( Marlin \& Allen 1999, Ferraz et al. 2010).

Um estudo realizado por Ferraz et al. (2010), indicou que os cavalos participantes de uma partida treino de polo, considerado alto handicap, foram submetidos a um esforço de alta intensidade com evidência de alterações nos eletrólitos e equilíbrio ácido-base de potencial relevância. Neste contexto, para cavalos de enduro, Holbrook et al. (2006) afirmaram que alterações metabólicas sistêmicas podem, direta ou indiretamente, acarretar alterações da função cardiovascular, comprometendo a integridade miocárdica.

No que diz respeito à demanda cardiovascular, Marlin \& Allen (1999) observaram que em cavalos de polo, durante um Chukker, a média da frequência cardíaca atingida durante o esforço físico foi de $215 \mathrm{bpm}$. Este resultado esteve, juntamente com o observado em cavalos de corrida (210 - 220 bpm) (Evans 2009), entre os mais altos alcançados entre as modalidades esportivas equestres. Em contrapartida, para cavalos de salto foi documentado valores de 190 bpm (Stadler et al. 1993), para cavalos de adestramento de 154 bpm (Stadler et al. 1993) e para cavalos treinados em esteira 163 bpm (Gehlen et al. 2005).

0 teste de esforço é usado para melhor reproduzir as condições de trabalho que podem causar sinais clínicos, sendo algumas dessas técnicas a avaliação ecocardiográfica pré e pós-exercício (Marr \& Bowen 2010). Neste sentido, em humanos (Neilan et al. 2006) e equinos (Young 2003) que executaram exercício prolongado, foi revelado evidências ecocardiográficas de disfunção cardíaca, sendo que este risco foi maior em atletas com baixo nível de treinamento. Desta forma o exercício prolongado extenuante tem sido associado com disfunção transitória do ventrículo esquerdo e foi denominada "fadiga cardíaca" (Welsh et al. 2005, Douglas et al. 1987).

Pouco se sabe sobre a influência do exercício de polo na função cardíaca de equinos saudáveis. Por isto e por tudo citado acima, o objetivo deste estudo foi identificar e avaliar, através da ecocardiografia, os efeitos morfológicos e funcionais cardíacos de equinos após o exercício de polo.

\section{MATERIAL E MÉTODOS}

A utilização dos animais para este estudo foi avaliada e aprovada pelo comitê de ética no uso animal do Instituto de Ciências Biológicas da Universidade de Brasília sob protocolo no 21359/2010. Sendo eles 27 equinos, dos quais 13 eram machos e 14 eram fêmeas, com idade variando de cinco a 15 anos, peso entre 393,5 e $497 \mathrm{~kg}$, altura entre 1,48 e 1,68m e sem raça definida. Todos os animais eram provenientes da Coudelaria de Rincão do exército brasileiro, portanto possuíam o mesmo padrão pré-estabelecido para um cavalo militar. Estes animais faziam parte do pelotão de polo do1ํo Regimento de cavalaria de guarda, e estavam submetidos ao mesmo manejo nutricional e treinamento atlético por no mínimo um ano. Não apresentavam nenhuma anormalidade ao exame clínico de rotina incluindo o auscultatório cardíaco e estavam dentro do padrão de sanidade exigido por uma equipe de oficiais veterinários os quais eram controlados uma vez ao mês.

Os equinos foram avaliados dentro de um período de treinamento regular mais intenso, visando à participação no Campeona- to do Exército de polo/2010, sendo eles componentes da equipe do Comando Militar do Planalto considerados de baixo handicap (até oito gols). Este programa constituiu-se de quatro partidas treino semanal, sendo que cada animal jogou apenas um chukker por partida treino. Este treinamento iniciou-se no mês de junho e prorrogou-se até a véspera do campeonato, que ocorreu do dia 22 a 25 de julho, totalizando aproximadamente 30 dias. Assim, todas as avaliações ocorreram no período seco com umidade relativa do ar de aproximadamente $43 \%$ e temperatura ambiente média de $25^{\circ} \mathrm{C}$.

Foram realizadas duas avaliações ecocardiográficas para cada indivíduo em diferentes momentos. A primeira avaliação foi realizada em repouso, no dia em que os animais não foram treinados, ou seja, não realizaram exercício físico por no mínimo 24 horas. Este momento, portanto, foi caracterizado como momento zero $\left(\mathrm{M}_{0}\right)$. A segunda avaliação ocorreu dentro do período de cinco a dez minutos após o animal ter jogado um chukker, caracterizando assim o momento final $\left(\mathrm{M}_{\mathrm{f}}\right)$. Foram avaliados de cinco a sete animais por partida treino em um total de cinco eventos ao longo de duas semanas.

O exame ecocardiográfico procedeu-se no modo M pela janela paraesternal direita, obtendo-se as medidas de diâmetro interno do ventrículo esquerdo em sístole (DVES) e diástole (DVED), septo interventricular em sístole (SIVS) e diástole (SIVD), parede livre do ventrículo esquerdo em sístole (PLVES) e em diástole (PLVED), seguindo as orientações da Sociedade Americana de Ecocardiografia (Reef 1990). Para tanto, foi utilizado o aparelho de ecocardiografia modelo Sonosite Elite Plus $®$, com transdutor convexo banda larga de 2 a $4 \mathrm{MHz} 2 \mathrm{com}$ alcance de $25 \mathrm{~cm}$ de profundidade.

A fração de encurtamento (FE) foi calculada a partir dos valores obtidos do DVEd e DVEs através da fórmula: FE=[(DVEd-DVEs)/DVEd] x 100 (Rovira \& Muñoz 2009). Assim como a fração de ejeção (FEj) foi obtida pela fórmula [(VDVE-VSVE)/VDVE] x100. 0 volume sistólico final do ventrículo esquerdo (VSVE) e volume diastólico final do ventrículo esquerdo (VDVE) foram determinados pela fórmula modificada de Teicholz: [7 x (DVE) ${ }^{3}$ / 2,4 + DVE] (Teicholz et al. 1976, Rovira \& Muñoz 2009). Por fim o volume sistólico (VS) foi obtido pela diferença entre VDVE e VSVE e o débito cardíaco (DC) foi obtido multiplicando-se o VS pela frequência cardíaca (FC) (Rovira \& Muñoz 2009).

Para avaliação da perda de peso corporal, foi utilizada uma balança portátil com plataforma adaptada (TOLEDO MGR-3000 Júnior®). Os dados em repouso foram obtidos no mesmo período em que foi realizada a primeira avaliação ecocardiográfica $\left(\mathrm{M}_{0}\right)$. Os valores após o exercício foram obtidos ao final da partida treino $\left(\mathrm{M}_{\mathrm{f}}\right)$, quando todos os animais avaliados nesta ocasião já estivessem sem os acessórios de montaria, sendo que até o momento da pesagem não tiveram acesso a água ou qualquer tipo de alimento.

Os dados em repouso e após o exercício foram submetidos à análise descritiva, buscando assim a obtenção dos valores relativos à média e desvio padrão. Em seguida, estes foram submetidos ao teste de normalidade de Kolmogorov-Smirnov e ao tratamento estatístico por meio da aplicação do teste "U" de Mann-Whitney, com nível de significância de 5\% comparando-se os dois momentos.

\section{RESULTADOS}

A média e desvio padrão dos índices funcionais cardíacos, obtidos por meio da ecocardiografia, e do peso corporal dos animais empregados neste estudo, foram expressos no Quadro 1. 
Quadro 1. Representação dos índices ecocardiográficos e peso corporal (média \pm desvio padrão) de equinos de polo em repouso $\left(M_{0}\right)$ e após exercício $\left(M_{f}\right)$

\begin{tabular}{lcc}
\hline \multicolumn{1}{c}{ Variáveis } & $\mathrm{M}_{0}$ & $\mathrm{M}_{\mathrm{f}}$ \\
\hline SIVs (cm) & $4,28 \pm 0,35^{*}$ & $4,06 \pm 0,40^{*}$ \\
DVEs (cm) & $5,91 \pm 0,81^{*}$ & $6,66 \pm 0,75^{*}$ \\
PLVEs (cm) & $3,74 \pm 0,51^{*}$ & $2,96 \pm 0,51^{*}$ \\
SIVd (cm) & $3,15 \pm 0,35^{*}$ & $2,87 \pm 0,28^{*}$ \\
DVEd (cm) & $9,74 \pm 0,86$ & $9,60 \pm 0,15$ \\
PLVEd (cm) & $2,60 \pm 0,51^{*}$ & $1,80 \pm 0,32^{*}$ \\
DC (l/min) & $78,14 \pm 29,16^{*}$ & $133,86 \pm 38,71^{*}$ \\
VS (ml) & $2139,27 \pm 556,21^{*}$ & $1722,41 \pm 487,66^{*}$ \\
FE (\%) & $39,45 \pm 5,29^{*}$ & $30,58 \pm 5,58^{*}$ \\
FEj (\%) & $77,06 \pm 6,29^{*}$ & $65,43 \pm 8,22^{*}$ \\
FC (bpm) & $35,70 \pm 6,27^{*}$ & $77,41 \pm 15,42^{*}$ \\
Peso (Kg) & $426,96 \pm 29,13$ & $420,48 \pm 32,10$
\end{tabular}

Valores com sobrescritos* em uma mesma linha são estatisticamente diferentes. Espessura do septo interventricular em sístole (SIVs); diâmetro interno do ventrículo esquerdo em sístole (DVEs); espessura da parede livre do ventrículo esquerdo em sístole (PLVEs); Espessura do septo interventricular em diástole (SIVd); diâmetro interno do ventrículo esquerdo em diástole (DVEd); espessura da parede livre do ventrículo esquerdo em diástole (PLVEd); débito cardíaco (DC); volume sistólico (VS); fração de encurtamento (FE); fração de ejeção (FEj); frequência cardíaca (FC).

\section{DISCUSSÃO}

\section{Índices morfológicos}

0 exercício de polo reduziu a espessura do miocárdio (SIVd, SIVs, PLVEd, PLVEs). Esse achado corroborou com o documentado por Gehlen et al. (2005) ao estudarem cavalos de concurso completo de equitação (CCE), para os quais, tanto os animais treinados como os não treinados apresentaram redução destes índices quando avaliados dentro de dois minutos após exercício em esteira. Schefer et al. (2010) também observaram redução significante da PLVEs cinco minutos após os cavalos terem sido exercitados em esteira rolante. Em contrapartida, Michima (2007) e Bello et al. (2011) que avaliaram equinos após competição de enduro, encontraram aumento da espessura do SIV e nenhuma alteração para a PLVE. Este achado foi observado por Michima (2007) principalmente em animais desclassificados por causas metabólicas e por Bello et al. (2011) nos cavalos finalistas que percorreram $120 \mathrm{~km}$ e não nos animais que realizaram percursos menores. Ambos os autores coletaram os dados no período igual ou superior a 30 minutos.

De acordo com o proposto por Marr e Bowen (2010) a contratilidade do miocárdio pode ter sido comprometida após o exercício de polo, pois a mesma deveria aumentar com a estimulação simpática induzida pelo exercício causando aumento da espessura do SIV e PLVE durante a sístole. A resposta do miocárdio representado pela espessura do SIV e PLVE após o exercício demonstrou ser variável entre os autores que retrataram diferentes modalidades de esforço (Gehlen et al. 2005, Michima 2007, Bello et al. 2009, Schefer et al. 2010), sugerindo que estas alterações poderiam estar relacionadas com o tipo de exercício e/ou o período de avaliação.

O DVE aumentou quando em sístole (Quadro 1) de forma similar ao observado por Gehlen at al. (2005), que encontraram aumento do DVEd em cavalos treinados para CCE, $\mathrm{e}$ para ambos os índices (DVEd e DVEs), em cavalos destreinados avaliados logo após exercício em esteira. Trivax et al. (2010) associaram o aumento do diâmetro do ventrículo direito após o exercício ao aumento da pré e pós-carga. Por outro lado, para Michima (2007) e Bello et al. (2011) houve diminuição significante do DVEs e DVEd de equinos após exercício de enduro e foi associada a distúrbios metabólicos e/ou desidratação. Para Di Bello et al. (1996) dependendo do tipo de exercício, duração e intensidade de carga cardíaca, diferentes mudanças na dimensão cardíaca após o exercício foram descritas em humanos.

Trivax et al. (2010), ao avaliarem a função cardíaca direita, constataram em humanos que cerca de $1 / 3$ dos corredores bem treinados apresentaram evidências de disfunção, com dilatação significativa do átrio e do ventrículo direito, e hipocinesia do ventrículo direito imediatamente após completarem uma maratona. Além disso, para Trivax et al. (2010), a maioria desses atletas, demonstraram uma redução significativa da FEj do ventrículo direito e evidências bioquímicas de mionecrose cardíaca incluindo um pequeno aumento transitório de troponina cardíaca I. No presente estudo, observamos resultado semelhante referente o ventrículo esquerdo, ou seja, aumento DVEs, hipocinesia de PLVE em determinados animais e redução significativa da FEj. Contudo, não avaliamos marcadores bioquímicos de lesão cardíaca e não encontramos outros estudos que mencionassem estes achados para cavalos de polo, logo não foi possível estabelecer se houve injúria cardíaca ou se foi uma condição transitória específica da modalidade.

\section{Índices funcionais}

O DC aumentou de forma significativa $(194,21 \%)$, isto ocorreu, pois segundo o proposto por Marr (1999), esse dado mostrou-se dependente da pré-carga, e sua elevação foi observada em situações associadas ao maior retorno venoso e menor resistência periférica, como ocorre durante o exercício. Para Hinchcliff et al. (2008), este aumento ocorreu devido à combinação da policitemia induzida pela contração esplênica com o incremento da $\mathrm{FC}$, e uma menor contribuição do VS elevado. Segundo Boffi (2006) o aumento do fluxo sanguíneo foi diretamente relacionado com a intensidade do exercício. Este resultado sugeriu que, em média, não houve desidratação considerável nestes animais, pois de acordo com Naylor et al. (1993), em cavalos desidratados o DC diminuiu durante o exercício. A perda de peso, usada como uma forma representativa da perda de fluido corpóreo, também se mostrou insignificante reforçando esta ideia.

O VS diminuiu de forma significante após o exercício de polo coincidindo com o mencionado por Bello et al. (2011), quando observaram redução significante do VS nos cavalos de enduro que percorreram 90 e $120 \mathrm{~km}$. Para Dávila-Roman et al. (1997), a diminuição do volume sistólico no período imediatamente após o exercício indicou fadiga cardíaca por esforço. Da mesma forma, Kalliokoski et al. (2004) consideraram que alterações leves nos parâmetros de função ventricular esquerda, incluindo a redução do VS, foram sinais característicos de fadiga cardíaca em maratonistas humanos. 
Segundo Evans (2004), a fração de encurtamento (FE) fornece uma indicação da contratilidade cardíaca e de acordo com Douglas et al. (1987) a disfunção cardíaca reversível poderia estar relacionada à intensidade do exercício, pois a redução da FE tendeu a ser maior entre aqueles com os menores tempos durante a corrida. Indo ao encontro do nosso estudo com cavalos de polo, para Gehlen et al. (2005) e Schefer et al. (2010) retrataram uma redução significante deste índice em equinos após realizarem exercício em esteira. Para Bello et al. (2011) também houve uma redução significante da FE em cavalos de enduro que percorreram $90 \mathrm{~km}$, e concordaram com Kalliokoski et al. (2004) que foi um sinal de fadiga cardíaca. Para Marr e Bowen (2010), a mensuração da FE antes e imediatamente depois do exercício foi usada para tentar identificar doenças sutis do miocárdio que causam redução do desempenho atlético e que somente são visíveis após o exercício. Entretanto, estes autores ressaltaram que a diminuição da FE após o exercício também pode refletir as mudanças da pré-carga associada à redistribuição do fluxo sanguíneo após o exercício extenuante.

Outro método comum para avaliação da função sistólica foi à obtenção dos índices da fração de ejeção (FEj) ventricular, que representou o desempenho ventricular esquerdo de um modo global. Coincidindo com nosso achado, uma análise quantitativa realizada com equinos após exercício em esteira, verificou-se que a FEj diminuiu significativamente após o exercício em comparação com os valores basais (Schefer et al. 2010). Rifai et al. (1999) também encontraram redução significante da FEj e movimentação anormal de parede em indivíduos humanos, e que, por sua correlação com aumento da troponina $\mathrm{T}$, sugeriram que estas anormalidades funcionais de VE poderia não ser apenas fadiga cardíaca, mas sim injúria. Entretanto, estudando atletas humanos após competição de triatlon, Douglas et al. (1987) relataram o retorno ao normal das alterações de movimentação de parede e FEj em 24 a 48 horas após o exercício, indicando que estes foram efeitos transitórios provocados pelo exercício.

De acordo com os resultados encontrados para VS, FE e FEj e os relatos acima, sugere-se que o exercício de polo demandou alta carga de atividade cardíaca e que possivelmente contribuiu para ocorrência de fadiga miocárdica transitória. De acordo com Douglas et al. (1987), esta condição pode assumir importância clínica em atletas que competem com frequência ou por muitos anos, em animais submetidos a competições de alto nível em condições ambientais extremas e ainda naqueles com disfunção cardíaca subjacente ou idosos.

Segundo Evans (2009), após o exercício a FC diminuiu rapidamente no primeiro minuto, e depois continua a diminuir de forma mais lenta, sendo que a taxa de recuperação da FC foi mais rápida em cavalos treinados. Para este autor, a FC após o exercício pode ser usada como guia para recuperação inadequada, pois se mantivesse acima de $130 \mathrm{bpm}$ no período de cinco a dez minutos após o exercício indicaria que o animal não foi treinado o suficiente, ou poderia possuir desordens clínicas como fibrilação atrial, infecção respiratória ou claudicação. Portanto, sendo 77 bpm a mé- dia da FC dos cavalos de polo deste estudo após um período de cinco a 10 minutos de recuperação, demonstrou que, de acordo com Evans (2009), não houve superaquecimento e o condicionamento foi adequado.

A perda de peso corpóreo foi em média de 1,52\% após um chukker, sem diferença estatística entre o $\mathrm{M}_{0}$ e $\mathrm{M}_{\mathrm{f}}$. Isto sugere que estes animais não sofreram desidratação significante visto que, para Kingston et al. (1997), a mensuração da perda de peso corporal seria uma forma confiável de se estimar a perda de fluidos por meio do suor em equinos exercitados. De outra forma Teixeira-Neto (2006) e Dumont et al. (2010) encontraram perda de peso corporal de 2,4\% e de 4,30 a $5,27 \%$ respectivamente, ao avaliarem cavalos após exercício de enduro sugerindo que a desidratação tem maior influência em cavalos que realizam enduro a polo.

\section{CONCLUSÕES}

Com o presente estudo demonstramos que o esforço físico requerido pela modalidade polo provocou diversas alterações na função cardíaca de equinos saudáveis, comprovadas através da ecocardiografia. Os resultados sugeriram alta demanda cardíaca com capacidade de deprimir a função ventricular esquerda no período de cinco a dez minutos após o esforço. A partir destes resultados, torna-se apropriado a inclusão de uma avaliação de triagem para que os animais iniciem a atividade atlética da modalidade polo com segurança. Defini-se ainda que deva ser estabelecido um programa de treinamento que vise ao condicionamento cardiovascular. Novos estudos são necessários para acompanhar o tempo necessário de recuperação cardíaca, definir se estas alterações são acompanhadas de lesão miocárdica e identificar o que este tipo de esforço pode provocar em longo prazo tanto na função cardíaca como em sua estrutura.

Agradecimentos.- Ao Conselho Nacional de Desenvolvimento Científico e Tecnológico (CNPq) pelo apoio financeiro e ao 10 Regimento de Cavalaria de Guarda e todos que ali contribuíram gentilmente com a realização deste trabalho.

\section{REFERÊNCIAS}

Bello C.A.O., Vasconcelos C.E.S., Godoy R.F., Teixeira Neto A.R., \& Lima E.M.M. 2011. Ecocardiografia de equinos Puro Sangue Árabe após exercício de enduro de diferentes intensidades. Cienc. Rural. 41(1):132136.

Boffi F.M. 2006. Fisiologia del Ejercicio em Equinos. Buenos Aires, Inter-médica. 306p.

Dávila-Román V.G., Guest T.M., Tuteur P.G., Rowe W.J., Ladenson J.H. \& Jaffe A.S. 1997.Transient right but not left ventricular dysfunction after strenuous exercise at high altitude. Journal of the American College of Cardiology. 30(2):468-473.

Di Bello V., Santoro G., Talarico L., Di Muro C. \& Caputo M.T. 1996. Left ventricular function during exercise in athletes and sedentary men. Medicine and Science in Sports and Exercise. 28:190-196.

Douglas P.S., O’toole M.L., Hiller W.D., Hackney K. \& Reichek N. 1987. Cardiac fatigue after prolonged exercise. Circulation 76:1206-1213.

Dumont C.B., Leite C.R., Moraes J.M., Alves R.O., Godoy R.F. \& Lima E.M.M. 2010. Parâmetros eletrocardiográficos de equinos Puro Sangue Árabe submetidos a exercício prolongado de enduro. Cienc. Rural 40:19661973.

Evans D. 2004. Exercise testing in the field, p.9-18. In: Hinchcliff K.W., Ka- 
neps A.J. \& Geor R.J. (Eds), Equine Sports Medicine and Surgery. W.B. Saunders, London.

Evans D.L. 2009. Cardiovacular physiology: Responses to exercise and training. I Simpósio de Fisiologia de Exercício em Equinos, São Paulo, p.4-6. (Resumo de palestras)

Ferraz G.C., Soares O.A.B., Foz N.S., Carvalho M.P. \& Queiroz-Neto A. 2010. The workload and plasma ion concentration in a training match session of high-goal (elite) polo ponies. Equine Vet. J. 42:191-195.

Gehlen H., Silke M., Karl R., Franz E. \& Peter S. 2005. Echocardiographic comparison of left ventricular dimensions and function after standardized treadmill exercise in trained and untrained healthy warmblood horses. Equine Comp. Exercise Physiol. 3(1):3-11.

Hinchcliff K.W., Geor R.J. \& Kaneps A.J. 2008. Equine exercise physiology: The science of exercise in the athletic horse. W.B. Saunders, London, p.221-235.

Holbrook T., Birks E.K., Sleeper M.M. \& Durando M. 2006. Endurance exercise is associated with increased plasma cardiac troponin I in horses. Equine Vet. J. 36(Suppl.):27-31.

Kalliokoski K.K., Laaksonen M.S., Loutolahti M., Laine H., Takala T.O., Nuutila P. \& Knuuti J. 2004. Myocardial perfusion after marathon running. Scand. J. Med. Sci. Sports 14(1):208-214.

Kingston J.K., Geor R.J. \& McCutcheon L.J. 1997. Use of dew point hygrometry, direct sweat collection and measurement of body water losses to determine sweating rates in exercising horses. Equine Vet. J. 22(Suppl.):108-113.

Marlin D. \& Allen J. 1999.Cardiovascular demands of competition on low-goal (non-elite) polo ponies. Equine Vet. J. 31(Suppl.):378-382.

Marr C.M., Bright J.M., Marlin D.J., Harris P.A. \& Roberts C.A. 1999. Pre- and post- exercise echocardiography in horses performing treadmill exercise in cool and hot/humid conditions. Equine Vet. J. 30(Suppl.):131-136.

Marr C.M. \& Bowen I.M. 2010. Cardiology of the Horse. $2^{\text {nd }}$ ed. W.B. Saunders, London, p.294.

Michima L.E.S. 2007. Influência do exercício físico prolongado sobre a concentração sérica de troponina I cardíaca e sobre a função cardíaca em cavalos de enduro. Tese de Doutorado em Clínica Veterinária, Faculdade de Ciências Agrárias e Medicina Veterinária, USP, São Paulo, SP. 94p.

Naylor J.R., Bayly W.M., Gollnick P.D., Brengelmann G.L. \& Hodgson D.R.
1993. Effects of dehydration on thermoregulatory responses of horse during low-intensity exercise. J. Appl. Physiol. 75:994-1001.

Neilan T.G., Danita M.Y., Pamela S.D., Jane E.M., Elkan F.H., David L., Michael H.P. \& Malissa J.W. 2006. Among nonelite participants in the Boston marathon myocardial injury and ventricular dysfunction related to training levels. Circulation 114:2325-2333.

Reef V.B. 1990. Echocardiographic examination in the horse: The basics. Compend. Contin. Educ. Vet. Pract. 12(9):312-319.

Rifai N., Douglas S.P., O’Toole M., Rimm E. \& Ginsburg G.S. 1999. Cardiac troponin $\mathrm{T}$ and I, electrocardiographic wall motion analyses, and ejection fractions in athletes participating in the Hawaii ironman triathlon. Am. J. Cardiol. 83:1085-1089.

Rovira S. \& Muñoz A. 2009. Two-dimensional- and M-Mode echocardiographic measurements and indices of cardiac function in Spanish Colts and fillies of different age. J. Vet. Med. Sci. 71(7):957-964.

Schefer K.D., Bitschnau C., Weishaupt M.A. \& Schwarzwald C.C. 2010. Quantitative analysis of stress echocardiograms in healthy horses with 2-dimensional (2d) echocardiography, anatomical m-mode, tissue doppler imaging, and 2d speckle tracking. J. Vet. Intern. Med. 24:918-931.

Stadler P., Rewel A. \& Deegen E. 1993. Die M mode echocardiography in dressage- and showjumping horses of class ' $\mathrm{S}$ ' and in untrained horses. J. Vet. Med. 40:292-306.

Teicholz L.E., Kreulen T. \& Herman M.V. 1976. Problems in echocardiographic volume determinations: Echocardiographic-angiographic correlations in the presence or absence of synergy. Am. J. Cardio. 37:7-11.

Teixeira Neto A.R. 2006. Variáveis fisiológicas e estresse oxidativo de equinos durante campeonato de enduro. Tese de Doutorado em Medicina Veterinária, Faculdade de Ciências Agrárias e Veterinária, Universidade Estadual Paulista, Jaboticabal, SP. 84p.

Trivax J.E., Barry A.F., James A.G., Kavitha M.C., Michael J.G., Adam T.J., James M.C., David E.H. \& Peter A.M. 2010. Acute cardiac effects of marathon running. J. Appl. Physiol. 108:1148-1153.

Welsh R.C., Darren E.R.W., Dennis P.H., Dylan A.T., Jonathon M. \& Mark J.H. 2005. Prolonged strenuous exercise alters the cardiovascular response to dobutamine stimulation in male athletes. J. Physiol. 569(1):325-330.

Young L.E. 2003. Equine athletes, the equine atlhetes's heart and racing sucess. Exp. Physiol. 88:306-309. 